\title{
Back in the Sun
}

The recent suite of ground and space observatories bring solar physics into the twenty-first century. Solar Orbiter, due to launch this month, will observe the polar regions from up close, which is essential for understanding the magnetic field of the Sun.

$\mathrm{S}$ olar physics is an ancient science, dating back to 1300 BC in Babylon, with records of solar eclipses and, later in China, the addition of sunspot observations. Since then, numerous ground-based telescopes and space observatories have recorded the Sun's activities, yet fundamental questions regarding how its magnetic activity heats up the corona and accelerates the particles in the solar wind remain. As our modern technology is vulnerable to the coronal mass ejections and solar flares that adversely affect our electrical and communications systems on Earth, it's become more pressing to understand and predict solar activity.

In February 2020, the ESA-led Solar Orbiter will launch from Cape Canaveral, USA, and head for the innermost heliosphere around the Sun. It will get to within 42 million $\mathrm{km}(0.28 \mathrm{au})$ of the Sun in a highly elliptic orbit (with aphelion $1 \mathrm{au}$ ). Crucially, its orbital plane will be tilted with respect to the solar plane, so that the on-board instruments will be able to observe the Sun from latitudes between $\pm 25^{\circ}$. During the extended mission it may be possible to reach latitudes in excess of $30^{\circ}$. It will be the first time that we will be able to observe the polar regions of the Sun close up, and peer into a coronal hole - these are cool (therefore dark) regions of open magnetic field lines from which solar wind escapes at high speed.

During closest approach, Solar Orbiter's speed will roughly match the Sun's rotation speed, allowing it to hover - for a few days - above the same patch of the Sun in order to monitor flares and eruptions from the build-up of magnetic fields in the atmosphere. The ten instruments (or suites of instruments) are divided into 'remote sensing' and 'in situ' instruments. The remote sensing ones will monitor the dynamics and surface of the Sun over a wide wavelength range, while the in situ ones will focus on the particles, plasma waves and fields in the solar wind directly above the regions studied by the remote sensing instruments.

As described by Daniel Müller and co-workers in this issue's Mission Control, the main science objectives are to find out (1) what drives the solar wind and from where the coronal magnetic field originates, (2) how solar transients drive changes in the heliosphere, (3) how solar eruptions lead to energetic particle radiation throughout the

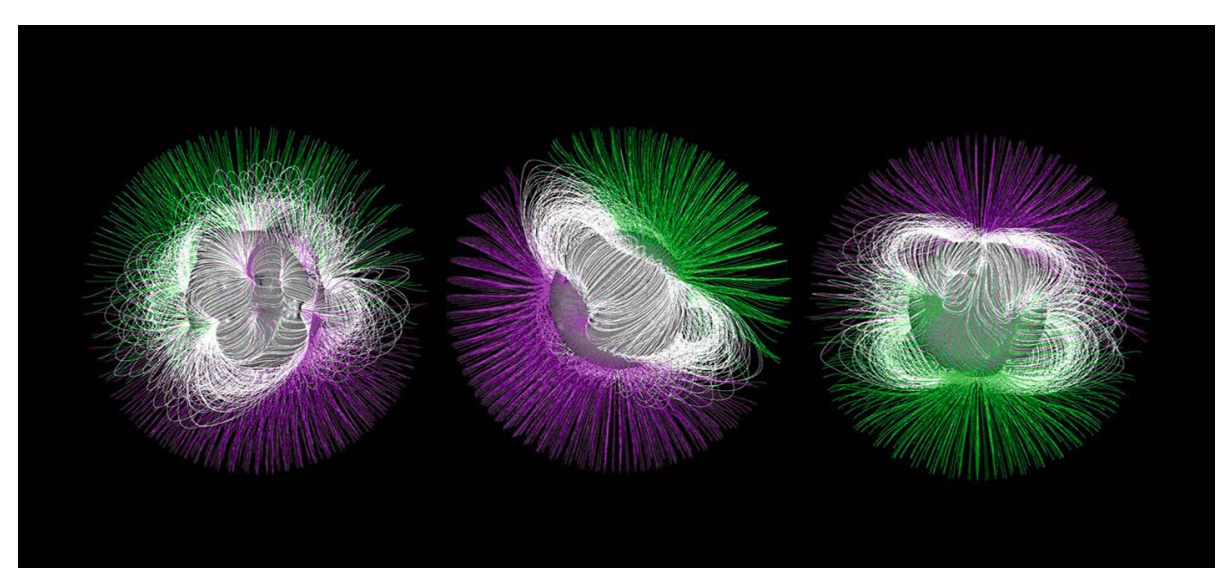

Credit: NASA Goddard Space Flight Center

heliosphere, and (4) how the solar dynamo works, including how it connects the Sun and the heliosphere.

Solar Orbiter will also work in conjunction with NASA's Parker Solar Probe (PSP), which launched in August 2018 and is already 'listening' to solar wind up close, within half of Mercury's orbit. PSP will be sampling the solar corona and measuring solar wind during 24 perihelion passes, eventually getting to within nine solar radii of the solar surface (closer than Solar Orbiter). Adam Szabo provides a brief overview in his Mission Control from September 2018. In his News \& Views, Eugene Parker describes the preliminary results from the first two completed orbits; already, PSP has revealed a "dynamic, young and unevolved solar wind unlike that seen at 1 au."

Besides Solar Orbiter and PSP, a number of other ground-based and space observatories are busy monitoring solar activity. On Earth the largest solar observatory is also the newest: the Daniel K. Inouye Solar Telescope (DKIST) on Maui, Hawai' $i$, which released its spectacular first image of the Sun on 29 January. From July 2020 it will focus on magnetic fields at the Sun's surface and into the outer atmosphere, recording details three times smaller than previously resolvable. It will also detect energetic particles in the solar wind plasma, and hopefully help to uncover the pathway between the smallest magnetic structures to large-scale terrestrial and interplanetary plasmas.

Since 1995, the Solar and Heliospheric Observatory (SOHO) has been in a halo orbit around the first Lagrangian point (L1) between the Earth and the Sun, continuously measuring properties of the solar interior, the corona and the solar wind over a wide frequency band. The mission has been extended numerous times, and is likely to continue until at least 2022 (to be determined later in 2020). Its long lifespan has meant that SOHO has covered an entire solar cycle of approximately 22 years, during which the Sun's polarity changes by $360^{\circ}$, which helped to understand the (negligible) role of the Sun on climate change, for instance. The magnetic field lines (pictured) are shown at 1997 (solar minimum), 2002 and 2013 (both solar maxima with opposite polarity) from left to right, where green is positive polarity and magenta negative.

While many other missions exist, focusing on the solar corona (Hinode), coronal mass ejections (Solar Terrestrial Relations Observatory), magnetic field generation and structure (Solar Dynamics Observatory), lower solar atmosphere (Interface Region Imaging Spectrograph) and more, they were mostly launched over a decade ago. With Solar Orbiter, PSP and DKIST, we are on the cusp of a transformation of our understanding of the Sun. Not only will we find answers to some long-standing mysteries, we will undoubtedly come up with questions we hadn't thought to ask before. That is scientific progress.

Published online: 12 February 2020 https://doi.org/10.1038/s41550-020-1032-4 